\title{
Dislocation Characterization by the Direct-fitting/modified Williamson-Hall (DF/mWH) Method in Cold Worked Ferritic Steel
}

\author{
Setsuo TAKAKI, ${ }^{1)}$ Takuro MASUMURA ${ }^{1 / *}$ and Toshihiro TSUCHIYAMA ${ }^{1,2)}$ \\ 1) Research Center for Steel, Kyushu University, 744 Moto-oka, Nishi-ku, Fukuoka, 819-0395 Japan. \\ 2) Department of Materials Science and Engineering, Kyushu University, 744 Moto-oka, Nishi-ku, Fukuoka, $819-0395$ Japan.
}

(Received on September 10, 2018; accepted on October 25, 2018)

\begin{abstract}
X-ray diffraction is a powerful tool for dislocation characterization, which includes evaluation of dislocation distribution, nature of dislocation, and dislocation density. In the Williamson-Hall (WH) plots, the full width at half maximum (FWHM) is plotted relative to the diffraction angle for each diffraction peak and the method corresponds to the basic approach for dislocation characterization. However, the elastic anisotropy in each crystal plane makes the analysis of WH plots difficult because elastic anisotropy also affects the FWHM of diffraction peaks. In order to correct the effect of elastic anisotropy, Ungár developed a unique methodology by using the contrast factor $C$, and this is termed as the modified Williamson-Hall $(\mathrm{mWH})$ method. Conversely, researchers developed a new methodology termed as the "direct-fitting (DF) method," in which the elastic anisotropy is corrected by directly applying the diffraction Young's modulus ratio $(\omega)$. In the DF method, a linear relation is realized in the corrected WH plots, and reliable values are obtained for the parameter $\alpha$ that contains information on the crystallite size. In the present study, the $\alpha$-value obtained using the DF method was applied to the $\mathrm{mWH}$ equation, and dislocation characterization was performed in a low carbon ferritic steel (Fe-0.0056\%C) by cold rolling. The results indicated that increasing the extent of cold rolling decreases the screw component of dislocation, and monotonically increases the parameter $\varphi$ (which contains the information of dislocation density). Additionally, the parameter $A$ (which depends on the dislocation arrangement) was evaluated at approximately 0.50 for cold worked ferrite.
\end{abstract}

KEYWORDS: Williamson-Hall plots; direct-fitting/modified Williamson-Hall method; modified Williamson-Hall equation; elastic anisotropy; dislocation characterization; ferritic steel; work hardening.

\section{Introduction}

Work hardening is a useful technology to realize high strength in steel and it is known as "Bailey-Hirsch relation" wherein the increments in strength are proportional to the square root of dislocation density. ${ }^{1)}$ Dislocation density is identified by the total length of dislocations in unit volume, and thus it is directly measured by transmission electron microscopy when the dislocation density $\rho$ is not extremely high $\left(\rho<10^{14} / \mathrm{m}^{2}\right){ }^{2-5)}$ However, it is not easy to apply the methodology using radiation diffraction in highly dislocated materials to estimate dislocation density. Williamson proposed a basic approach to evaluate the micro-strain $\varepsilon$ that is produced by dislocations. When X-rays with a wave length $\lambda$ are used for diffraction analysis and a diffraction angle $\theta$ and full width at half maximum (FWHM) $\beta$ is obtained in each diffraction peak, the following Williamson-Hall (WH) equation is constructed as a function of parameter $K$ $(=2 \sin \theta / \lambda)$ and $\Delta K(=\beta \cos \theta / \lambda)^{6)}$ as follows:

$$
\Delta K=\alpha+\varepsilon K
$$

Here, the parameter $\alpha$ is dependent on the crystallite

* Corresponding author: E-mail: masumura@zaiko.kyushu-u.ac.jp DOI: https://doi.org/10.2355/isijinternational.ISIJINT-2018-623 size. In poly crystalline metals, several diffraction peaks appear and $K$ and $\Delta K$ is obtained from each diffraction peak. Therefore, the values of parameter $\alpha$ and $\varepsilon$ are determined based on the relation between $K$ and $\Delta K$. However, a linear relation is not typically obtained due to elastic anisotropy in each crystal plane $\{\mathrm{hkl}\}$.

Generally, elastic behavior is discussed in terms of the orientation parameter $\Gamma$ that is expressed by the following equation as a function of the Miller index $\{\mathrm{hkl}\}$ as follows:

$$
\Gamma=\left(\mathrm{h}^{2} \mathrm{k}^{2}+\mathrm{k}^{2} \mathrm{l}^{2}+\mathrm{l}^{2} \mathrm{~h}^{2}\right) /\left(\mathrm{h}^{2}+\mathrm{k}^{2}+\mathrm{l}^{2}\right)^{2} \quad(0 \leqq \Gamma \leqq 1 / 3)
$$

In the case of bcc-Fe, the $\Gamma$-value corresponds to 0 for $\{200\}, 0.09$ for $\{310\}, 0.25$ for $\{110\}$ and $\{211\}$, and $1 / 3$ for $\{222\}$. Ungár proposed a method to correct the elastic anisotropy in WH plots by using the contrast factor $C$ that is given by the following equation: ${ }^{7)}$

$$
C=C_{\mathrm{h} 00}(1-q \Gamma)
$$

where $C_{\mathrm{h} 00}$ denotes the contrast factor in the crystal plane $\{\mathrm{h} 00\}$ and $q$ denotes a constant, which depends on the screw component of dislocation $S(0 \leqq S \leqq 1)$. This is expressed as follows:

$$
C_{\mathrm{h} 00}=(1-S) C_{\mathrm{h} 00}^{E}+S C_{\mathrm{h} 00}^{S}
$$




$$
q=(1-S) q^{E}+S q^{S}
$$

Symbols $\mathrm{E}$ and $\mathrm{S}$ denote edge dislocation and screw dislocation, respectively. In the case of bcc-Fe, $C_{\mathrm{h} 00}^{E}=$ $0.256, C_{\mathrm{h} 00}^{S}=0.305, q^{E}=1.310$, and $q^{S}=2.647 .{ }^{8)}$ Ungár reconstructed the WH equation as follows by applying the contrast factor $C$. Plots of $\Delta K$ vs. $K \sqrt{C}$ are termed as modified Williamson-Hall $(\mathrm{mWH})$ plots. The expression is as follows:

$$
\Delta K=\alpha+\varphi K \sqrt{C}+O K^{2} C
$$

The authors confirmed that the third term on the righthand side of the equation is negligibly low when compared with the values of other terms, ${ }^{9}$ and thus the above equation is simplified as follows:

$$
\Delta K=\alpha+\varphi K \sqrt{C}
$$

Here, $\varphi$ denotes the parameter that contains the information on dislocation density $\rho$ and dislocation distribution $A$, and it is expressed by the following equation as functions of $\rho, A$, and the magnitude of Burgers vector of dislocation $b$ :

$$
\varphi=(\pi / 2)^{1 / 2} A b \sqrt{\rho}
$$

We combine Eqs. (3) and (7) to obtain the following equation ( $\mathrm{mWH}$ equation):

$$
(\Delta K-\alpha)^{2} / K^{2}=\varphi^{2} C_{\mathrm{h} 00}(1-q \Gamma)
$$

If a reliable value is obtained for the parameter $\alpha$, then the values of $(\Delta K-\alpha)^{2} / K^{2}(=Y)$ are identically determined for each diffraction peak. In the modified Williamson-Hall method, the value of parameter $\alpha$ is determined to yield the optimal fit in the $Y$ vs. $\Gamma$ plots. The plots of $Y$ vs. $\Gamma$ should display a linear relation, and thus the $q$-value is estimated from the slope of the plots. Based on the determined $q$-value, the screw component $S$ is obtained from Eq. (5). The value of parameter $\varphi$ is also estimated from the value $Y_{0}$ at $\Gamma=0$ as follows:

$$
\varphi=\left(Y_{0} / C_{\mathrm{h} 00}\right)^{1 / 2}
$$

Conversely, authors reported that the elastic anisotropy in WH plots is precisely corrected by the parameter $\omega$, which is identified as follows:

$$
\omega=E_{\mathrm{hkl}}^{*} / E_{0}
$$

where $E_{\text {hkl }}^{*}$ denotes the diffraction Young's modulus and $E_{0}$ denotes the standard Young's modulus. ${ }^{10)}$ If the values of $E^{*}{ }_{\text {hkl }}$ and $E_{0}$ are known, then the values of $\omega$ are obtained for each diffraction peak. However, the aforementioned values are typically unknown, and thus the authors developed a new methodology termed as the "direct-fitting (DF) method" to correct the elastic anisotropy in WH plots. ${ }^{10)}$ The details of the DF method are not elucidated in the present study. However, it is confirmed that the elastic anisotropy in WH plots is accurately corrected by applying the optimal $\omega$ values to the following equation: ${ }^{10}$

$$
\Delta K=\alpha+\varepsilon(K / \omega)
$$

Based on the corrected WH plots of $\Delta K$ vs. $(K / \omega)$, we can obtain an accurate value for the parameter $\alpha$ as well as micro-strain $\varepsilon$.
In the study, cold rolled ferritic steel (Fe-0.0056\%C) was examined via X-ray diffraction analysis to investigate dislocation characteristics such as dislocation distribution, nature of dislocation, and dislocation density. First, the $\alpha$-value was obtained using the DF method, and it was then applied in Eq. (9) to determine the values of parameter $q$ and $\varphi$. Here, the process is termed "direct-Fitting/modified Williamson-Hall (DF/mWH) method". Subsequently, the values of parameter $A$ are estimated by the following equation that is reconstructed from Eq. (8):

$$
A \fallingdotseq 0.798 \varphi /(b \sqrt{\rho})
$$

The values of dislocation density $\rho$ were already measured by the modified Williamson-Hall/Warren-Averbach (mWH/WA) method in a previous study, ${ }^{11)}$ and thus they are applied to determine parameter $A$ in Eq. (13).

\section{Experimental Procedure}

The material used in the investigation corresponds to a well-annealed low carbon ferritic steel $(\mathrm{Fe}-0.0056 \% \mathrm{C})$ with an average grain size of approximately $50 \mu \mathrm{m}$. The results confirmed that the annealed material exhibits random crystal orientation. Steel sheets with thickness ranging from $1.1 \mathrm{~mm}$ to $5 \mathrm{~mm}$ were cold rolled to a thickness of $1 \mathrm{~mm}$ and then subjected to X-ray diffraction analysis by utilizing $\mathrm{Cu}-\mathrm{K}_{\alpha}$ (wavelength: $0.15418 \mathrm{~nm}$ ). Specimens with a size of $15^{1} \times 15^{\mathrm{w}} \times 1^{\mathrm{t}} \mathrm{mm}$ were cut from the cold rolled steel sheets and then the surface of specimens was grinded using sand papers to obtain a flat surface. Subsequently, the surface of specimens was removed by $50 \mu \mathrm{m}$ by electrolytic polishing to remove the surface layer affected by the sand paper grinding. ${ }^{12)}$ The rotation speed of detector was $0.003 \mathrm{deg} / \mathrm{s}$. The instrumental function was corrected based on Voigt profiles analysis ${ }^{13)}$ using the standard material: $\mathrm{LaB}_{6}$, (No.: SRM660c produced by NIST). The fitting index that shows the accuracy of data fitting was evaluated by the square of the Correl function. The tensile test was conducted on the JIS-13B specimens to measure the yield stress of cold rolled specimens at room temperature at a cross-head speed of 1 $\mathrm{mm} / \mathrm{min}$.

\section{Experimental Results and Discussion}

\subsection{Determination of Parameter $\alpha$ by the Direct-Fitting Method}

Figure 1 shows the WH plots in $60 \%$ cold rolled specimen where FWHM is extremely high and the reliability of the analyzed result is high. The Young's modulus is extremely low in $\langle 200\rangle$ and $<310\rangle$ direction such that $\Delta K$ is high in $\{200\}$ and $\{310\}$ plane. Conversely, $\Delta K$ is low in $\{222\}$ plane because it exhibits high Young's modulus. The crystal planes corresponding to $\{110\}$ and $\{211\}$ exhibit middle values with respect to Young's modulus. Given this type of an elastic anisotropy, the WH plots are characterized by irregular arrangement. In order to correct the aforementioned type of elastic anisotropy, the optimal values were determined for parameter $\omega$ by the DF method. ${ }^{10)}$ The obtained results are listed in Table 1 with the values of $K$ and $\Delta K$. 


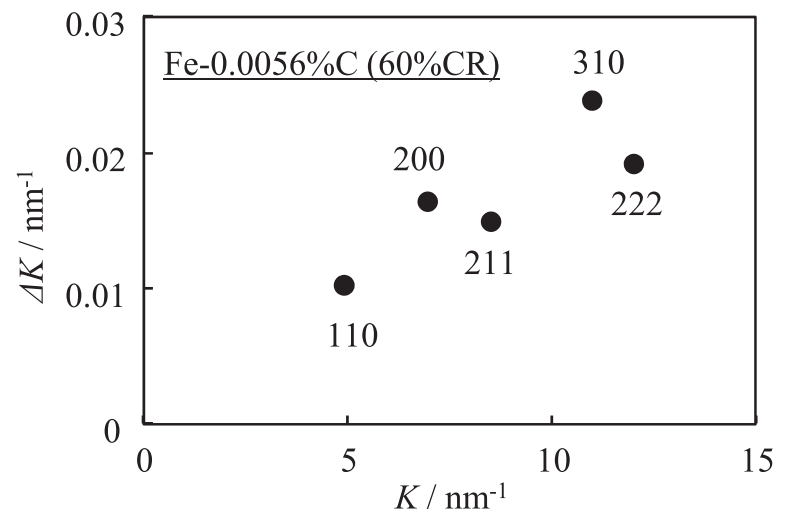

Fig. 1. Williamson-Hall plots in the $60 \%$ cold rolled specimen.

Table 1. Values of $K, \Delta K$, and parameter $\omega_{\mathrm{hkl}}$ obtained by the direct fitting method in the $60 \%$ cold rolled specimen.

\begin{tabular}{ccccc}
\hline $\mathrm{hkl}$ & $K\left(\mathrm{~nm}^{-1}\right)$ & $\Delta K\left(\mathrm{~nm}^{-1}\right)$ & $\omega_{\text {hkl }}$ & $K / \omega_{\text {hkl }}$ \\
\hline 110 & 4.93 & 0.01015 & 0.985 & 5.01 \\
200 & 6.97 & 0.01637 & 0.738 & 9.45 \\
211 & 8.54 & 0.01478 & 0.985 & 8.67 \\
310 & 11.03 & 0.02379 & 0.812 & 13.59 \\
222 & 12.08 & 0.01912 & 1.109 & 10.89 \\
\hline
\end{tabular}

The corrected WH plots of $\Delta K$ vs $(K / \omega)$ are shown in Fig. 2. The results indicate that a linear relation is realized in the corrected WH plots. In this case, the value of parameter $\alpha$ was estimated as $0.0016 \mathrm{~nm}^{-1}$. Here, it should be noted that the reliability of data is also verified by the DF method. The scattering of corrected WH plots is mainly attributed to the measurement error corresponding to $\beta$ in the parameter $\Delta K$. The fitting index decreases with increases in the measurement error $\beta$. The maximization of the fitting index is desirable. In the case of Fig. 2, the fitting index is extremely high and reaches 0.989 , thereby implying that the reliability of the used data is extremely high.

\subsection{Determination of Parameters $q$ and $\varphi$ by the Modi- fied Williamson-Hall Equation}

In order to estimate the parameter $q$, the $\alpha$-value $(0.0016$ $\mathrm{nm}^{-1}$ ) obtained by the DF method is input in the left term of Eq. (9). The values of $(\Delta K-0.0016)^{2} / K^{2}$ are calculated for each $\{\mathrm{hkl}\}$ plane by applying the data in Table 1 , and thus they are plotted as a function of the orientation parameter $\Gamma$ as shown in Fig. 3. Based on the slope, the $q$-value is estimated as 1.621. We substitute $q=1.621$ in Eq. (5), and parameter $S$ is determined as 0.233 . We substitute $S=0.233$ in Eq. (4), and contrast factor $C_{\mathrm{h} 00}$ is determined as 0.267 . Given that $Y_{0}=4.574 \times 10^{-6}$, parameter $\varphi$ is calculated as 0.00414 from Eq. (10). It is noted that all parameters are simply obtained by the DF/mWH method.

In order to reconfirm the reliability of the $\varphi$-value (0.00414), the mWH plots are obtained by using the above results. The $\mathrm{mWH}$ plots are shown in Fig. 4. Good linearity is realized between $K \sqrt{C}$ and $\Delta K$, and the slope yields the value of 0.00414 for parameter $\varphi$. The value is completely identical to that obtained by the $\mathrm{DF} / \mathrm{mWH}$ method. The result supports the reasonability of the $\mathrm{DF} / \mathrm{mWH}$ method.

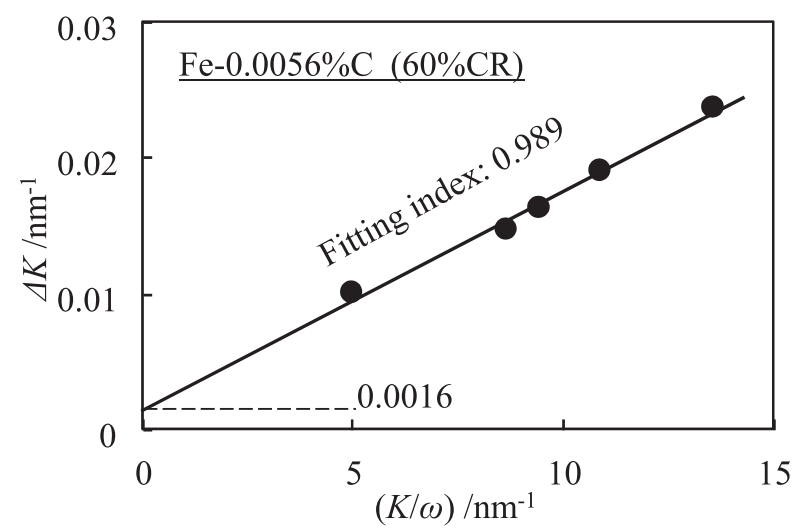

Fig. 2. Corrected Williamson-Hall plots in the $60 \%$ cold rolled specimen.

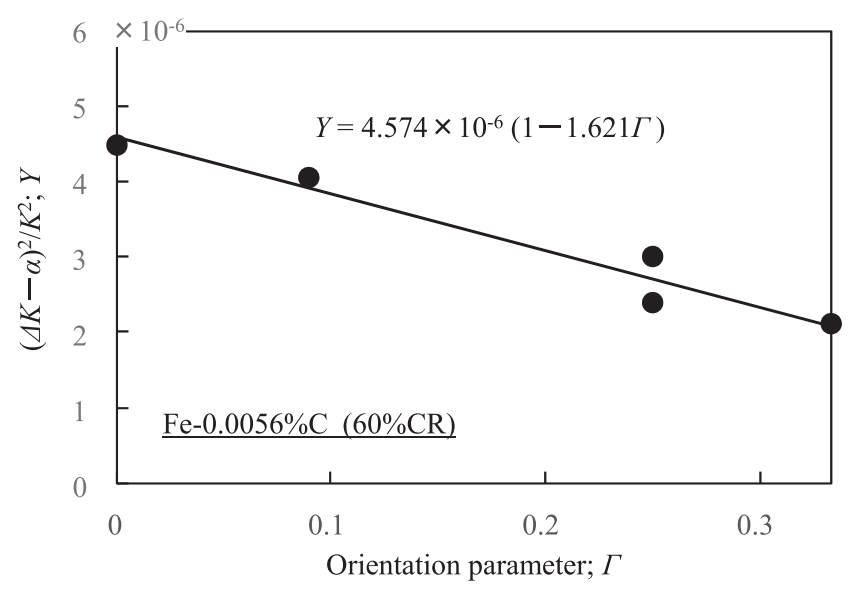

Fig. 3. Relation between the orientation parameter $\Gamma$ and value of $(\Delta K-\alpha)^{2} / K^{2}$ in the $60 \%$ cold rolled specimen.

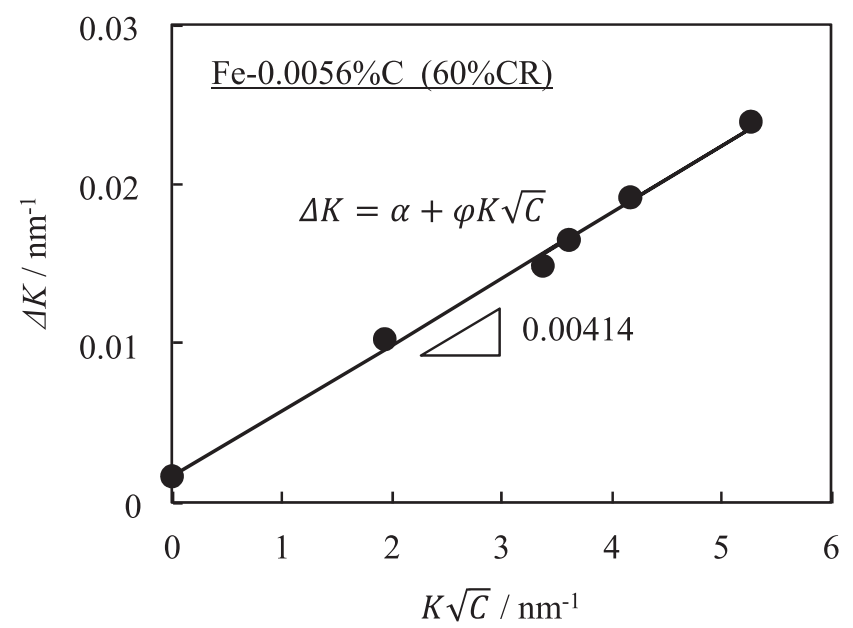

Fig. 4. Modified Williamson-Hall plots in the $60 \%$ cold rolled specimen.

Additionally, if the dislocation density is already known, Eq. (13) yields the value for parameter $A$. In the case of the $60 \%$ cold rolled specimen, the dislocation density is estimated at $5.35 \times 10^{14} / \mathrm{m}^{2}$ by the $\mathrm{mWH} / \mathrm{WA}$ method, ${ }^{11)}$ and thus parameter $A$ corresponds to 0.483 in Eq. (13). In the next section, the changes in the aforementioned parameters are discussed with respect to the amount of cold working. 


\subsection{Changes of Parameters with Cold Rolling}

In this section, the $\mathrm{DF} / \mathrm{mWH}$ method is applied to several specimens with cold rolling of up to $80 \%$ thickness reduction. First, the values of parameter $\alpha$ are shown in Fig. 5. The two or three plots at each rolling reduction simply correspond to the results of repeatability measurement. The $\alpha$-value is in the range from 0.001 to $0.005 \mathrm{~nm}^{-1}$ and barely changes with increases in the amount of cold rolling. The fitting index in the DF method exceeded 0.98 in each specimen used in Fig. 5.

As mentioned above, parameter $q$ is obtained by inputting $\alpha$-value in Eq. (9). The values of parameter $q$ are plotted in Fig. 6. The $q$-value decreases with increases in the amount of cold rolling. This implies that the screw component decreases in the stored dislocations with cold working. An extant study reported that the recovery of dislocations is activated when dislocation density exceeds a critical value, namely $2 \times 10^{14} / \mathrm{m}^{2}{ }^{14)}$ It is widely known that the recovery of dislocations mainly occurs through the cross slip of screw dislocations and the dislocation reaction, thereby leading to the disappearance of screw dislocations. Additionally, after the cross slip of screw dislocations, a pair of edge dislocations remain on the cross-slip plane. This suggests that the ratio of screw dislocation decreases through the recovery

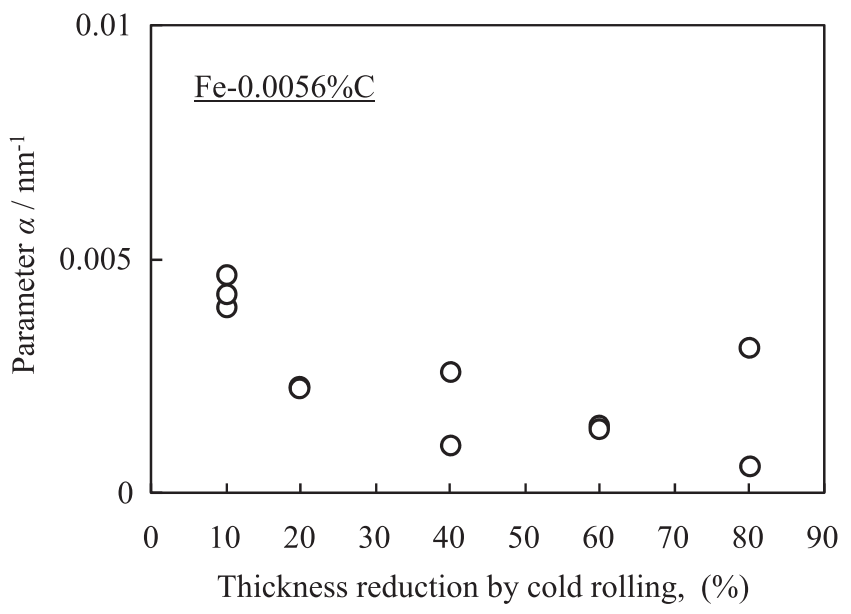

Fig. 5. Values of parameter $\alpha$ that are obtained by the direct-fitting method.

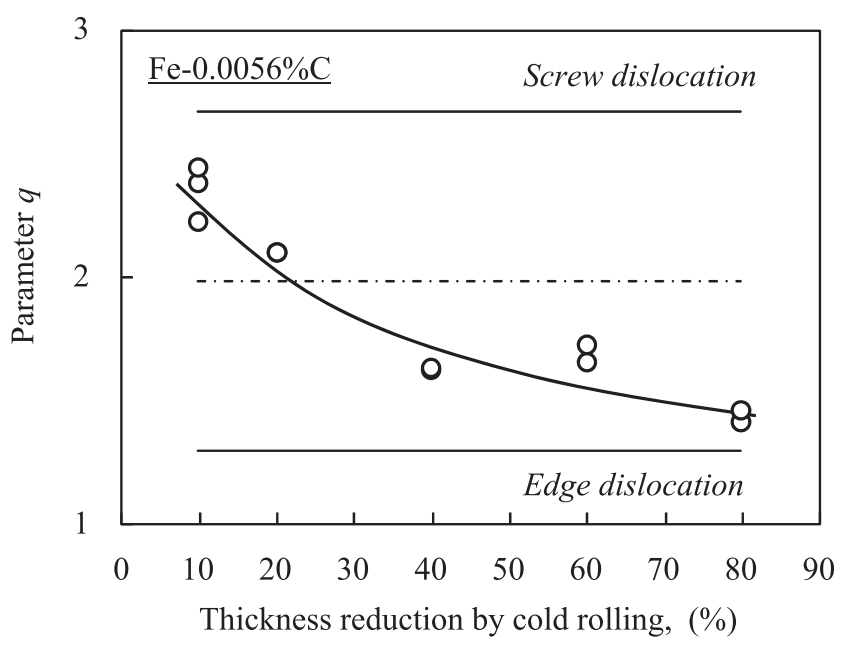

Fig. 6. Values of parameter $q$ that are obtained by the direct-fitting/ modified Williamson-Hall method. process of dislocations. Further examination is required to explain the phenomenon although it appears that the recovery of dislocations is related to the change in the screw component in the stored dislocations.

Additionally, the value of parameter $\varphi$ is obtained by the $\mathrm{DF} / \mathrm{mWH}$ method as well as the $q$-value. Figure 7 shows the change in the $\varphi$-value with cold rolling. The $\varphi$-value increases with increases in the amount of cold rolling. The $\varphi$-value contains information on the dislocation density, and thus it should be discussed in connection with work hardening behavior. Figure $\mathbf{8}$ shows the relation between yield stress $\sigma_{y}$ and the $\varphi$-value obtained in several cold rolled specimens. Although a few errors exist in the $10 \%$ cold rolled specimens, the results indicated that the linear relation holds between $\sigma_{y}$ and $\varphi$ and is expressed as follows:

$$
\Delta \sigma[\mathrm{GPa}]=113 \times \varphi \text {. }
$$

In the case of the $10 \%$ cold rolled specimen, the value of FWHM in diffraction peaks is extremely low such that there are a few errors with respect to the estimated $\varphi$-values. The $\varphi$-value is proportional to $\sqrt{\rho}$ as shown in Eq. (8), and thus the result in Fig. 8 suggests the Bailey-Hirsh relationship with respect to the yield stress of cold rolled specimens.

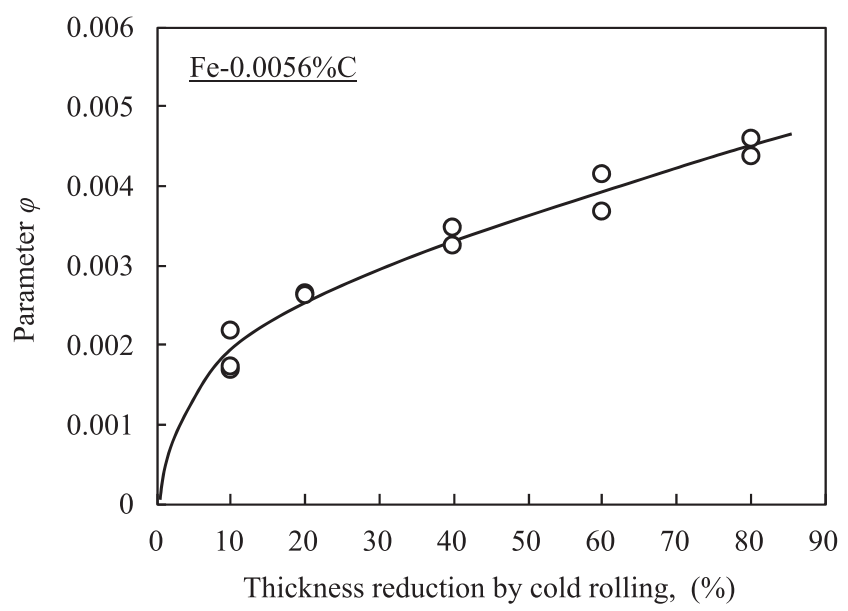

Fig. 7. Values of parameter $\varphi$ that are obtained by the direct-fitting/ modified Williamson-Hall method.

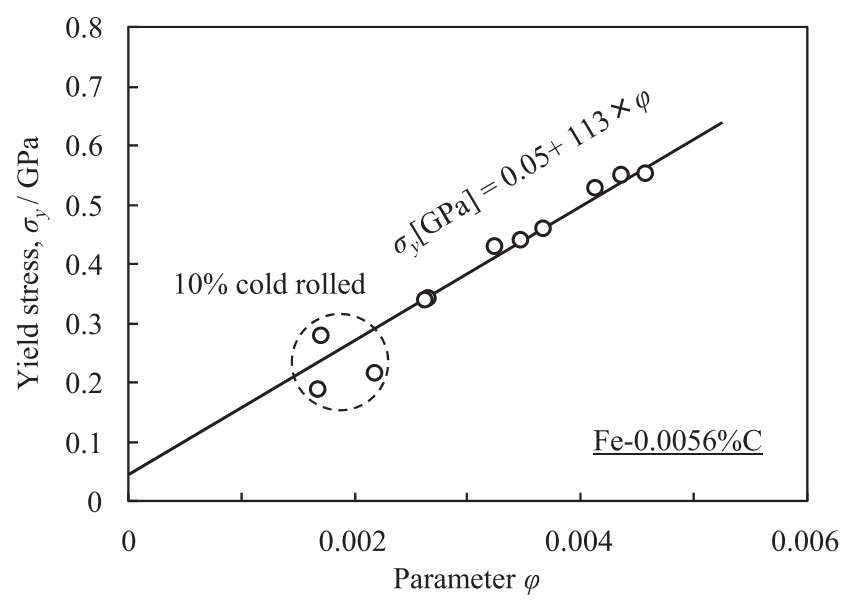

Fig. 8. Relation between yield stress and $\varphi$-value that is obtained by the direct-fitting/modified Williamson-Hall method in the cold rolled specimens. 


\subsection{Estimation of the Parameter $\boldsymbol{A}$ in Cold Worked Ferritic Steel}

In a previous study, ${ }^{11}$ the dislocation density of cold rolled specimens was measured by the mWH/WA method, and the Bailey-Hirsch relation between dislocation density $\rho$ and yield stress $\sigma_{y}$ was reported. The result is shown again in Fig. 9. In the figure, the results reported by other studies $^{2-5)}$ are also plotted. The results indicate that all data are almost on the line expressed by the following BaileyHirsch equation:

$$
\sigma_{y}[\mathrm{GPa}]=0.05+18 \sqrt{\rho} / 10^{9} .
$$

Additionally, an extant study reported that grain size affects the behaviors of dislocation introduction as well as work hardening for ferritic steels, and thus the aforementioned Bailey-Hirsch relation holds for yield stress irrespective of grain size. ${ }^{14)}$ Chemical composition also affects the behavior of dislocation introduction. For example, Fig. 10 shows the effect of carbon content on the Bailey-Hirsch relation that was constructed using the data reported by Goldman. ${ }^{4)}$ The amount of tensile deformation is identical in $0.04 \% \mathrm{C}$ steel and decarburized steel (approximately 1-12\% elongation). Goldman suggested that work hardening in

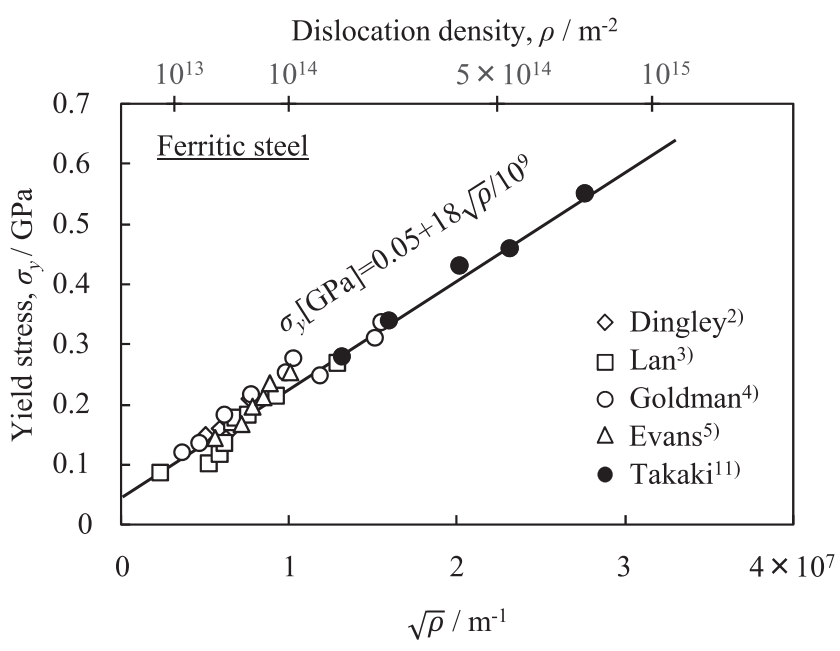

Fig. 9. Bailey-Hirsch relation in cold worked ferritic steels. ${ }^{11)}$

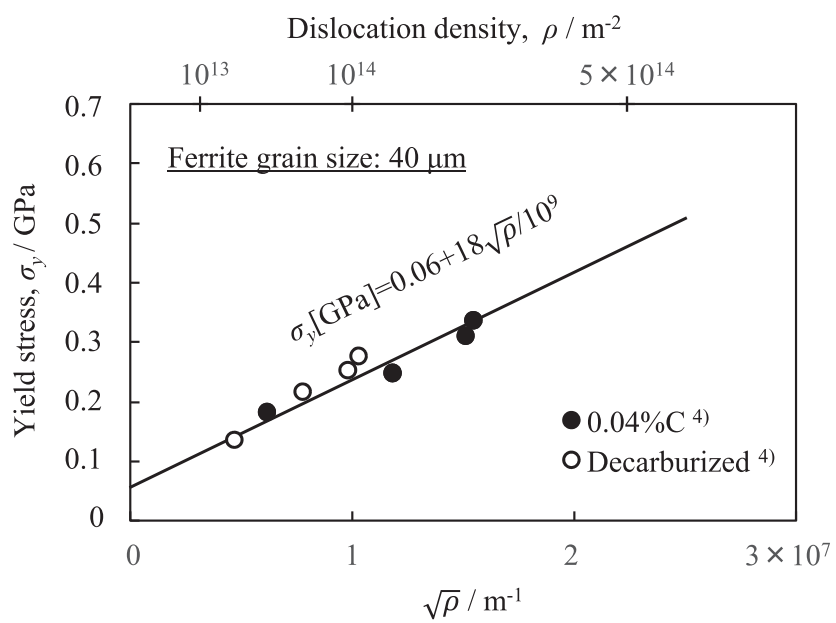

Fig. 10. Effect of carbon content on the Bailey-Hirsch relation in tensile-deformed ferritic steels with a grain size of 40 $\mu \mathrm{m}$.
$0.04 \% \mathrm{C}$ steel exceeds that in decarburized steel although the dislocation density is higher in the former even at the same percentage of deformation. It is interesting that all data are around the same line in which the strengthening coefficient is identical as shown in Eq. (15). The friction stress (0.05 GPa in Eq. (15)) varied based on testing temperature, strain rate, and chemical composition of steels although the strengthening coefficient appears identical irrespective of the chemical composition and ferrite grain size. This implies that the amount of work hardening $\Delta \sigma[\mathrm{Pa}]$ is expressed identically for ferritic steel by the following equation as a function of dislocation density $\rho\left[\mathrm{m}^{-2}\right]$ :

$$
\Delta \sigma=18 \sqrt{\rho} .
$$

Thus, the dislocation density of cold rolled ferritic steels is estimated by the following equation that is re-expressed based on Eq. (16) as follows:

$$
\rho=(\Delta \sigma / 18)^{2} .
$$

Table 2 shows the values of dislocation density that are calculated from the yield stress of cold rolled specimens.

Based on the results of Fig. 7 and Table 2, the parameter $A$ is introduced by substituting the values of $\rho$ and $\varphi$ into Eq. (13). The calculated results are shown in Fig. 11. The calculation error for the $10 \%$ cold rolled specimen is slightly high although the result corresponding to $A=0.5$ is obtained in the other specimens. The result indicates that $\mathrm{A} \fallingdotseq 0.5$ is applied for cold worked ferritic steel irrespective of the amount of deformation and that the dislocation density is estimated from parameter $\varphi$ by the following equation:

$$
\rho=2 \varphi^{2} /\left(\pi A^{2} b^{2}\right) \quad(b=0.25 \mathrm{~nm} \text { for } \mathrm{Fe}) .
$$

In order to verify the reliability of the above equation, the

Table 2. Dislocation density of cold rolled specimens that is calculated from the increment of yield stress $\Delta \sigma$ $\left(=\sigma_{y}-0.05\right)$

\begin{tabular}{cccccc}
\hline Cold rolling $(\%)$ & 10 & 20 & 40 & 60 & 80 \\
\hline$\sigma_{y}(\mathrm{GPa})$ & 0.28 & 0.34 & 0.43 & 0.46 & 0.55 \\
$\Delta \sigma(\mathrm{GPa})$ & 0.23 & 0.29 & 0.38 & 0.41 & 0.50 \\
$\rho\left(\times 10^{14} \mathrm{~m}^{-2}\right)$ & 1.63 & 2.60 & 4.46 & 5.19 & 7.72 \\
\hline
\end{tabular}

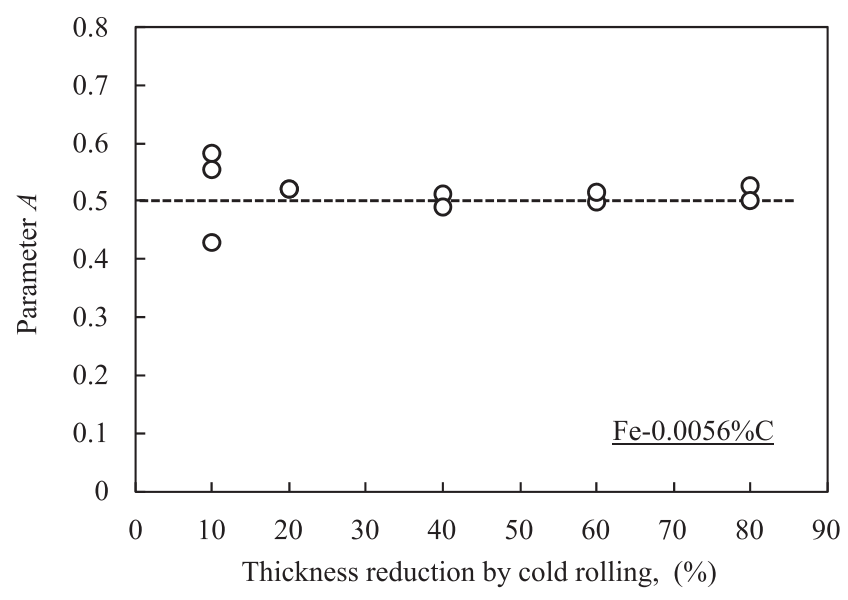

Fig. 11. Values of parameter $A$ that are calculated from the relation between dislocation density and parameter $\varphi$. 


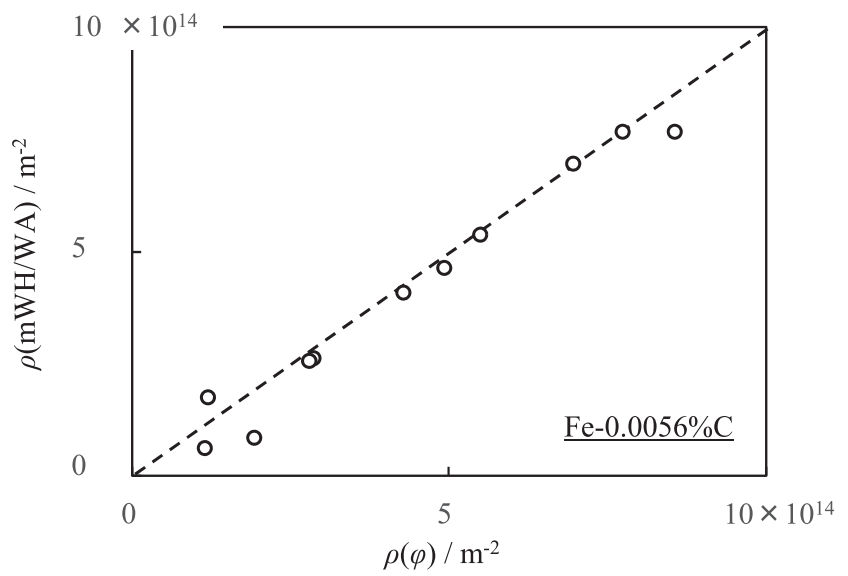

Fig. 12. Values of dislocation density that are obtained by the modified Williamson-Hall/Warren-Averbach method; $\rho(\mathrm{mWH} / \mathrm{WA})$ and estimated from parameter $\varphi$ by applying Eq. (18); $\rho(\varphi)$.

dislocation density is estimated by the $\mathrm{mWH} / \mathrm{WA}$ method for several cold rolled specimens and subsequently compared with the values calculated by Eq. (18). The result is shown in Fig. 12. The results confirm that a good correlation exists between the values obtained by the two different types of methods. Specifically, it should be noted that the value of parameter $A$ differs based on the type of materials (for e.g., martensitic steel and cold worked austenitic steel). However, when the value of parameter $A$ is obtained for the aforementioned metals, the same process is applied to estimate dislocation density.

\section{Conclusion}

The following conclusions were obtained in the study:

(1) The parameter $\alpha$ in the Williamson-Hall equation was accurately estimated by applying the direct-fitting method to Williamson-Hall plots.

(2) The $\alpha$-value was input in the modified Williamson-Hall equation, and the parameters $q$ and $\varphi$ were directly determined (direct-fitting/modified Williamson-Hall method).

(3) Dislocation density was estimated from the value of parameter $\varphi$ as follows:

$$
\rho=2 \varphi^{2} /\left(\pi A^{2} b^{2}\right)
$$

(4) With respect to parameter $A, A=0.5$ was obtained for cold worked ferrite irrespective of the amount of cold working.

\section{Acknowledgement}

This work was supported by JSPS KAKENHI Grant Number JP15H05768. This work was also partially supported by the Research Society for quantum-beam analysis of microstructures and properties of steels, ISIJ.

\section{REFERENCES}

1) J. E. Bailey and P. B. Hirsch: Philos. Mag., 5 (1960), 485

2) D. J. Dingley and D. MacLean: Acta Metall., 15 (1967), 885.

3) Y. Lan, H. J. Klaar and W. Dahl: Metall. Trans. A, 23 (1992), 545.

4) A. J. Goldman: Trans. Am. Soc. Met., 57 (1964), 900.

5) J. T. Evans and R. Rawlings: Mater. Sci. Eng., 4 (1969), 297.

6) G. K. Williamson and W. H. Hall: Acta Metall., 1 (1953), 22.

7) T. Ungár and A. Borbély: Appl. Phys. Lett., 69 (1996), 3173.

8) T. Ungár, J. Gubicza, G. Ribáric and A. Borbély: J. Appl. Crystal$\log r, 34$ (2001), 298.

9) S. Takaki, T. Masumura and T. Tsuchiyama: ISIJ Int., 58 (2018), 2354.

10) S. Takaki, F. Jiang, T. Masumura and T. Tsuchiyama: ISIJ Int., 58 (2018), 769.

11) S. Takaki, T. Masumura, F. Jiang and T. Tsuchiyama: Tetsu-toHagané, 104 (2018), 683 (in Japanese).

12) F. Jiang, K. Hirata, T. Masumura, T. Tsuchiyama and S. Takaki: ISIJ Int., 58 (2018), 376.

13) T. H. D. Keijser, J. I. Langford, E. J. Mittemeijer and A. B. P. Vogels: J. Appl. Crystallogr., 15 (1982), 308.

14) Y. Tanaka, S. Takaki, T. Tsuchiyama and R. Uemori: Tetsu-toHagané, 104 (2018), 284 (in Japanese). 